\title{
Mode locking and mode competition in a nonequilibrium solid-state condensate
}

\author{
P. R. Eastham \\ Blackett Laboratory, Imperial College London, London SW7 2BW, United Kingdom \\ (Received 20 March 2008; revised manuscript received 12 May 2008; published 21 July 2008)
}

\begin{abstract}
A trapped polariton condensate with continuous pumping and decay is analyzed using a generalized GrossPitaevskii model. Whereas an equilibrium condensate is characterized by a macroscopic occupation of a ground state, here the steady states take more general forms. Some are characterized by a large population in an excited state; others by large populations in several states, which may overlap spatially. In the latter case, the highly populated states synchronize to a common frequency above a critical density. At intermediate densities, they can drive condensation in other trap modes by parametric scattering. Estimates for the critical density of the synchronization transition are consistent with experiments.
\end{abstract}

DOI: 10.1103/PhysRevB.78.035319 PACS number(s): 71.36.+c, 03.75.Kk, 42.55.Sa, 71.35.Lk

\section{INTRODUCTION}

Recent experiments ${ }^{1-3}$ have provided substantial evidence for a new type of Bose-Einstein condensate, formed from polaritons in semiconductor microcavities. ${ }^{4}$ Although in many respects these results parallel those of condensation in atomic gases, the similarities conceal some fundamental differences. In particular, the lifetime of a polariton is typically only a few picoseconds and is less than the lifetime of the condensate. ${ }^{2}$ The condensate is therefore a nonequilibrium steady state in which the decaying polaritons are continually being replenished.

Several consequences of the nonequilibrium aspect of the system have now been predicted based on both microscopic calculations and generalizations of the Gross-Pitaevskii approach. ${ }^{5-10}$ While these predictions are undoubtedly interesting, more dramatic departures from the physics of equilibrium condensates are seen experimentally. An equilibrium condensate is characterized by a macroscopic occupation at the chemical potential; for a trapped ideal Bose gas, this is the ground-state energy of the trap, while more generally it is the lowest eigenvalue of the Gross-Pitaevskii equation. In some experiments, there is indeed evidence for equilibrated polariton distributions and large occupations of the $\vec{k}=0$ ground state of untrapped polaritons, ${ }^{1-3}$ but in others there are massive occupations associated with excited states. This has been observed in pillar traps, ${ }^{11}$ lattices, ${ }^{12}$ and disorder. ${ }^{13,14}$ Such results have been argued to be evidence of polariton lasing, ${ }^{11}$ distinct from condensation, but this distinction is clear only to the extent that condensation is restricted to equilibrium.

The aim of this paper is to outline a theory of the trapped polariton condensate with pumping and decay. The treatment is based on a recently proposed generalized Gross-Pitaevskii equation (gGPE). ${ }^{6,7}$ Motivated by the recent experiments, we go beyond the assumption ${ }^{5-8,15,16}$ of condensation at a single ground-state energy and systematically investigate how the dynamics of the pumped system can lead to more general forms. We first consider a small trap at low densities and show that the gGPE reduces to kinetic equations for the occupations of the trap states. The occupations of the states are determined by gain and loss processes which are independent of energy, i.e., a laserlike mode competition. Thus, the steady-state solutions can include massive occupations of an excited state or of several states simultaneously; the character of the steady states in this limit can be predicted from the wavefunctions of the trap.

Having established the steady-state structure at low densities, we then numerically explore how it changes away from this limit. We demonstrate that if the trap is such that there are several massively occupied states at low densities, then above a threshold density, the steady state reverts to a massive occupation at a single energy. This can be understood as the classical phenomenon of synchronization (mode locking) of coupled nonlinear oscillators. ${ }^{17}$ Supposing that the dominant nonlinearity is the polariton-polariton interaction, we predict that, for realistic parameters, polariton systems could be tuned through the synchronization transition. They thus give access to both an interaction dominated regime and a regime of laserlike mode competition.

A recent independent paper of Wouters ${ }^{18}$ discusses the synchronization of two nonequilibrium polariton condensates in a double-well trap. He found a threshold nonlinearity where the condensates lock to a single frequency, in general agreement with our results. However, that analysis assumes a double well with two separate regions for condensation. For such a trap, modeled on the usual Josephson weak link, there are no mode-competition effects. In contrast, we present an analysis of the low-density regime in an arbitrary trap. This analysis predicts that spatial separation is not needed to obtain coexisting condensates, in agreement with a recent experiment. ${ }^{13}$ Our numerical work then predicts synchronization between such spatially coexisting condensates with increasing density.

We consider the order-parameter equation, ${ }^{7}$

$$
i \partial_{t} \psi=\left\{H_{0}+U|\psi|^{2}+i\left[\gamma_{\mathrm{eff}}(r)-\Gamma|\psi|^{2}\right]\right\} \psi .
$$

This complex Ginzburg-Landau form has been argued ${ }^{6,7}$ to be the generic mean-field description of a polariton condensate with continuous pumping and decay. The first two terms on the right form the standard Gross-Pitaevskii mean-field equation, with local density $|\psi|^{2}$. $H_{0}$ includes a quadratic approximation to the polariton dispersion and a potential due to disorder or the trap. $U>0$ is the strength of the repulsive interaction between polaritons, which is treated as a contact interaction because its range is of the order of the exciton 
Bohr radius. The polarization degeneracy of the polaritons has been neglected for simplicity; it could be treated using a two-component order-parameter equation. ${ }^{15}$

The last two terms on the right of Eq. (1) account for the pumping and decay. The pumping model involves a reservoir of high-energy particles created by some external excitation. The condensate is populated by stimulated scattering from this reservoir, contributing a linear gain term $i \gamma(r) \psi$, where $\gamma(r)$ is related to the reservoir density. This term combines with a similar term from the decay of the polaritons, giving the overall linear gain term, with coefficient $\gamma_{\text {eff }}$, in Eq. (1). However, the rate of condensate growth should reduce with increasing density as the pump reservoir becomes depleted. This effect, modeled using a single gain-saturation coefficient $\Gamma$, gives the final term in Eq. (1). Physically, this form of pumping can be interpreted in terms of a pump which tries to locally enforce a steady-state density $\gamma_{\mathrm{eff}}(r) / \Gamma$.

\section{STRONG-TRAPPING LIMIT}

The steady states of Eq. (1) can be determined analytically in the limit of strong trapping, where the nonlinearities are weak compared with the single-particle level spacing. We may then treat them with degenerate perturbation theory. We expand $\psi(r, t)$ in terms of the eigenstates of $H_{0}, \psi_{n}^{0}$, and retain only the resonant terms in the resulting equations. To simplify the notation, we analyze a trap with only two singleparticle states, so $\psi(r, t)=\mu_{1}(t) \psi_{1}^{0}(r)+\mu_{2}(t) \psi_{2}^{0}(r)$, and assume homogeneous pumping. The amplitude $\mu_{1}(t)\left[\mu_{2}(t)\right]$ obeys

$$
\begin{aligned}
i \dot{\mu}_{1(2)}= & {\left[E_{1(2)}+i \gamma_{\mathrm{eff}}+(U-i \Gamma)\left(\eta_{1(2)}\left|\mu_{1(2)}\right|^{2}\right.\right.} \\
& \left.\left.+2 \beta\left|\mu_{2(1)}\right|^{2}\right)\right] \mu_{1(2)} .
\end{aligned}
$$

Here, $E_{i}$ is the single-particle energy, and the wavefunctions have been taken to be normalized and real. $\eta_{1}=u_{1111}, \eta_{2}$ $=u_{2222}$, and $\beta=u_{1122}$ are matrix elements for a local nonlinearity,

$$
u_{p p q q}=\int\left(\psi_{p}^{0}\right)^{2}\left(\psi_{q}^{0}\right)^{2} d r .
$$

They parametrize the inhomogeneous density profile of the trap states, with $\eta_{1}$ and $\eta_{2}$ describing the inhomogeneity of the states and $\beta$ their overlap.

Introducing number and phase variables in a rotating frame,

$$
\mu_{1(2)}(t)=e^{-i t \gamma_{\mathrm{eff}} U / \Gamma} \sqrt{n_{1(2)}(t)} e^{-i \phi_{1(2)}(t)},
$$

separates the number and phase dynamics. The former obeys the rate equations,

$$
\dot{n}_{1(2)}=2 \Gamma n_{1(2)}\left(\gamma_{\mathrm{eff}} / \Gamma-\eta_{1(2)} n_{1(2)}-2 \beta n_{2(1)}\right),
$$

with terms describing the stimulated scattering from the reservoir and the spontaneous decay, and the reservoir depletion. This result can be understood as a generalization of the kinetic description of polariton lasing ${ }^{19-22}$ to treat the spatial structure of the trap. It describes the extensive component of the occupation, and hence effects such as spontaneous pumping are missing. They would be important for a finite system close to the threshold. ${ }^{23}$
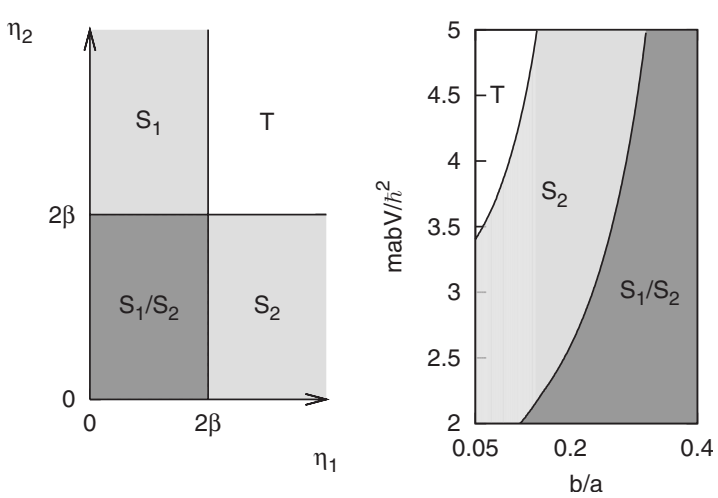

FIG. 1. Left panel: steady-state structure for condensation in a two-state trap for the generalized Gross-Pitaevskii model, neglecting nonresonant terms [Eq. (2)]. Lettering denotes the stable steady states in each region: condensation in both trap states $(T)$, state 1 alone $\left(S_{1}\right)$, or state 2 alone $\left(S_{2}\right)$. In the lower left region, both $S_{1}$ and $S_{2}$ are stable and the steady state is selected by the initial conditions. Right panel: steady-state structure for the two lowest states of a one-dimensional model trap, consisting of a hard-wall trap of length $a$ with a trap of length $b$ and depth $V$ at its center.

The phase dynamics is straightforward, obeying

$$
\dot{\phi}_{1(2)}=E_{1(2)}-U\left(\gamma_{\mathrm{eff}} / \Gamma-\eta_{1(2)} n_{1(2)}-2 \beta n_{2(1)}\right) .
$$

Each mode oscillates freely at a single-particle energy, which is shifted by the repulsive interactions.

Equation (5) has several steady-state solutions above the bulk condensation threshold $\gamma_{\text {eff }}=0$. There are always two solutions corresponding to condensation in each of the trap states: either $n_{1}^{s}=\gamma_{\text {eff }} /\left(\eta_{1} \Gamma\right)$ is finite and $n_{2}^{s}=0$ vanishes (state $S_{1}$ ), or vice versa (state $S_{2}$ ). However, if either $\eta_{1}, \eta_{2}>2 \beta$ or $\eta_{1}, \eta_{2}<2 \beta$, there is also a steady state $T$ with massive occupations of both trap states

$$
n_{1(2)}=\frac{\gamma_{\mathrm{eff}}}{\Gamma}\left(\frac{2 \beta-\eta_{2(1)}}{4 \beta^{2}-\eta_{1} \eta_{2}}\right) .
$$

The left panel of Fig. 1 shows the parameter regions in which the different steady states are stable. Linearizing Eq. (5) about the steady state $S_{1}$, we find that population fluctuations decay with rates $\lambda_{1}=2 \gamma$ and $\lambda_{2}=2 \gamma\left(2 \beta / \eta_{1}-1\right)$. Thus, this solution is stable for $\eta_{1}<2 \beta$. This is the condition that the occupation of the first trap state alone, determined by the pumping and the self-gain-saturation parameter $\eta_{1}$, is sufficient to keep the second below threshold. The analogous condition $\eta_{2}<2 \beta$ holds for the existence of a stable solution in which only the second trap state is occupied, $S_{2}$. If neither criterion is satisfied, both states must be occupied, and this is where the two-mode solution $T$ is stable-fluctuations there decay with rates $2 \gamma$ and $-2 \gamma\left(2 \beta-\eta_{1}\right)\left(2 \beta-\eta_{2}\right) /\left(4 \beta^{2}-\eta_{1} \eta_{2}\right)$.

The different steady-state solutions can be realized in many different potentials. For two widely separated states, we generically have $\beta \rightarrow 0$ and hence obtain two coexisting condensates. While this result is perhaps expected for separated traps, it can nonetheless also occur when there is substantial spatial overlap. A simple model which demonstrates this is a one-dimensional hard-wall trap of size $a$ with a finite 
trap of size $b$ and depth $V$ at its center. The right panel of Fig. 1 shows the different steady-state regions for the two lowest eigenstates of this potential.

Experimentally, the presence of massively occupied states in polariton systems is shown by bright luminescence peaks. The characteristic frequencies in such optical spectra follow from Eq. (6). In $S_{1}\left(S_{2}\right)$, the massively occupied states will lead to strong emission at the frequency $U \gamma_{\mathrm{eff}} / \Gamma+E_{1(2)}$, while in $T$ both peaks appear simultaneously. Note that here the blueshift of the condensing modes is $U \gamma_{\mathrm{eff}} / \Gamma$, irrespective of which steady state we consider. Physically, this is because the energy shifts are determined by the density, which is fixed by the pumping.

In addition to the strong emission associated with the condensing states, we also expect peaks in the optical response associated with the noncondensing trap states. These peaks can be understood as the confined quasiparticle excitations above the condensate. For the mean-field model (1) in the resonant approximation, these states are not populated and hence would appear in the absorption but not the luminescence. However, they could be populated by effects beyond that model, in which case they would appear weakly in luminescence. These peaks are shifted by the mean-field repulsion with the condensate, so in $S_{1}$ the absorption peak will be at $E_{2}+2 \beta U n_{1}$. Since its width will be proportional to $\lambda_{2}$, the mode is narrow close to the critical line $\eta_{1}=2 \beta$, where it goes unstable.

\section{BEYOND STRONG-TRAPPING}

Having established the physics of Eq. (1) in the strongtrapping regime, we now investigate how it develops with increasing nonlinearity. To do this we use real-space discretization to solve Eq. (1) directly for the one-dimensional model described above. This potential is chosen as a simple few-parameter model, which can be tuned into the different steady-state regions. We choose $a=m=\Gamma=1$, taking $a$ as the unit of length, $\hbar^{2} /\left(m a^{2}\right)$ as the unit of energy, and working with a rescaled density $|\psi|^{2} \rightarrow|\psi|^{2} / \Gamma$. Both the repulsive interaction $U$ and the gain saturation $\Gamma$ are believed to be significant for polaritons, ${ }^{1,6,7}$ and so for this demonstration, we take $U / \Gamma=1$.

Figure 2 illustrates how the steady-state spectra develop with increasing pumping. The analysis above, applied to the two lowest states of this potential, predicts the two-mode steady state $T$. This is in agreement with the results at weak pumping (lowest panel). Increasing the pumping, there is the overall blueshift associated with the increased density. The lowest two modes still dominate the spectrum, but their energy splitting has reduced slightly, and several further emission peaks appear (middle panel). Further increasing the pumping, the spectrum switches to emission at a single frequency (top panel).

These effects are due to the nonresonant terms dropped from Eq. (2). In a two-mode model with states of opposite parity, for example, there is the additional Josephson term

$$
\beta(U-i \Gamma) \mu_{1(2)}^{*} \mu_{2(1)} \mu_{2(1)}
$$

in the Eq. (2) for $\mu_{1}\left(\mu_{2}\right)$. Thus, the nonresonant terms couple together the phases of the condensing modes, leading to fre-
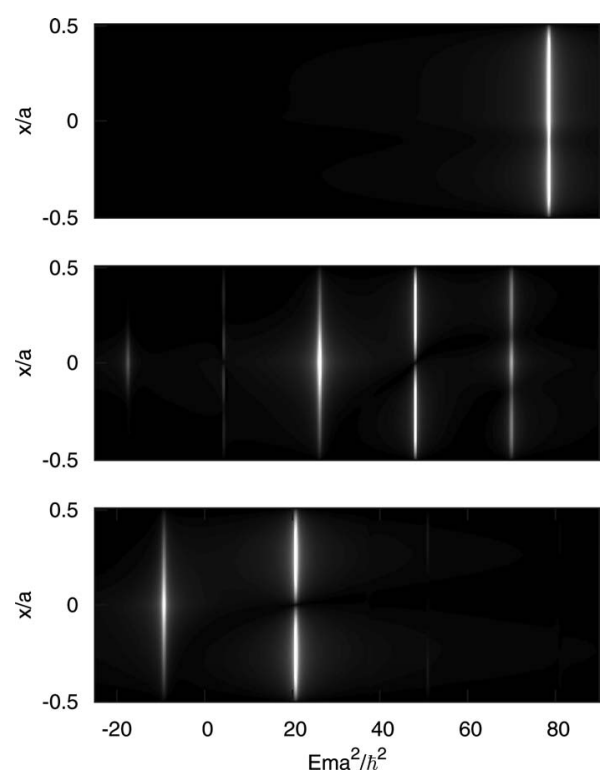

FIG. 2. Spectral analysis of the polariton field in the steady states of the generalized Gross-Pitaevskii model with the potential described in the caption of Fig. 1. The pump strength increases through $\gamma_{\mathrm{eff}}=1,30,60$ from the lowest panel to the highest. The grayscale is the computed amplitude $|\psi(E, x)|$, normalized by $\sqrt{\gamma_{\text {eff }}}$ to account for the overall increase in density. $U / \Gamma=1, b=0.05$ and $V=25$.

quency pulling and eventually synchronization. ${ }^{17}$ They also couple the phases of the condensed and uncondensed trap states, modifying the normal-mode frequencies of the latter. This can be understood as a renormalization of the quasiparticle spectrum by the condensate(s), which in the limit of an equilibrium, untrapped condensate leads to the usual Bogoliubov spectrum. Finally, we note that the nonresonant terms allow the condensing states to drive nonlinear emission at other frequencies. The highest energy line in the middle panel of Fig. 2, for example, can be interpreted as a condensate in the third trap state, generated by parametric scattering from the condensates in the two lowest trap states.

Coupled oscillators synchronize to a common frequency when the phase-phase couplings become comparable to their energy splitting $\Delta$. Since here the states have similar and overlapping density profiles, the matrix elements (3) are all of order one. The scale of the phase-dependent couplings, which would appear in Eq. (6), is then set only by the nonlinearity $\sqrt{U^{2}+\Gamma^{2}}$ and the polariton density $\rho \approx \gamma_{\mathrm{eff}} / \Gamma$. We may thus estimate the critical density $\rho_{c}$ or pump strength $\gamma_{c}$ from

$$
\Delta \sim \rho_{c} \sqrt{U^{2}+\Gamma^{2}} \sim \gamma_{\mathrm{c}} \sqrt{1+U^{2} / \Gamma^{2}} .
$$

This form is consistent with Fig. 2 and with simulation results (not shown) for other values of the nonlinearities $U$ and $\Gamma$. In particular, in the same model, the synchronization occurs between $\gamma_{\mathrm{eff}} / \Gamma=20$ and 40 when $U=0$, and between $U / \Gamma=20$ and 40 at fixed $\gamma_{\mathrm{eff}} / \Gamma=1$.

Synchronization due to nonlinear gain is well known as the basis for mode-locked lasers. However, an important difference between the polariton condensate and a laser is the 
presence of strong interactions $U$ between the particles due to the excitonic component of the polariton. Thus, frequency pulling and synchronization could be expected to have a much wider role in the physics of polariton condensates than they do in lasers, occurring on large energy scales at low intensities.

Let us estimate the critical polariton density $\rho_{c}$ for synchronization, supposing it is controlled by the real nonlinearity $U$. Estimates of this interaction are available for planewave excitons in a perfect quantum well and the localized exciton states of a disordered quantum well. ${ }^{24}$ For current experiments, a plausible upper limit is the result $U$ $\sim \Omega_{R}\left(m_{x} W\right)^{-1}$ from the disordered models, with disorder energy scale $W \sim 1 \mathrm{meV} . m_{x} \sim 0.5 m_{e}$ is the exciton mass and $\Omega_{R} \sim 20 \mathrm{meV}$ the Rabi splitting. Thus, the phase boundary for synchronization in a trap of scale $L_{t}$ is

$$
\Omega_{R}\left(m_{x} W\right)^{-1} \rho_{c} \sim 1 /\left(m L_{t}^{2}\right) .
$$

For a trap of $L_{t} \sim 5 \mu \mathrm{m}$ (Refs. 11 and 13) and a polariton mass $m \sim 10^{-5} m_{e}$, this gives $\rho_{c} \sim 10^{11} \mathrm{~cm}^{-2}$ or $n_{c} \sim L_{t}^{2} \rho_{c}$ $\sim 10^{3}$. This estimate is about one order of magnitude larger than the densities usually reported for polariton condensates. Thus, with a suitable potential, coexisting polariton condensates of different frequencies should be expected in tight traps. The synchronization transition could then be observed with increasing density, if necessary using larger traps to reduce $\rho_{c}$ into the experimentally accessible range.

Since we do not expect synchronization at current densities in tight traps, the present theory is consistent with the observations there of well-resolved emission lines. ${ }^{11,13}$ It also appears to be consistent with the existence of long-range coherence $^{1,14}$ at current densities with softer traps formed by disorder.

\section{CONCLUDING REMARKS}

Because the tightly trapped polariton condensate has wellresolved emission lines, it could provide a sensitive probe of the physics of nonequilibrium condensates. In particular, the present results will allow the order parameter Eq. (1) to be tested. While this form certainly captures much of the physics, ${ }^{6,7}$ effects that are important elsewhere are missing. Most obviously, there is no thermalization with the reservoirs, ${ }^{25,26}$ which exchange particles with the system irrespective of energy and do not directly cause transitions between trap states. Such effects would appear in generalizations of the kinetic Eq. (5). Since some groups ${ }^{1,2}$ report thermalized distributions, such generalizations may prove necessary.

To conclude, we have considered the trapped polariton condensate in the framework of a generalized GrossPitaevskii model. At low densities, this model admits solutions that, differently from an equilibrium condensate, involve massive occupations of excited states or of several states simultaneously. We have derived criteria for predicting the nature of the steady states in a given geometry and shown that the steady states are selected by gain-competition effects. At a general level, such physics is of course familiar in lasers, although it has not previously been considered for the polariton system. Moreover, the direct interactions between polaritons create differences compared with the photon laser: blueshifting the modes in the weak-nonlinearity regime and enhancing frequency pulling and synchronization in the strong-nonlinearity regime. Our estimates for the critical density of the synchronization transition suggest that it could be cleanly observed in tight traps ${ }^{11}$ and is likely to be responsible for the observations of long-range coherence across disorder potentials. ${ }^{1,14}$ This latter conclusion could be strengthened by extending the present work to include (i) a realistic model of the states in a disorder potential, (ii) thermalization processes, and (iii) fluctuations beyond the mean-field theory ${ }^{23}$ — which lead to linewidths ${ }^{13}$ for the condensing modes and hence compete with the synchronization.

\section{ACKNOWLEDGMENTS}

This work was supported by EPSRC-GB Grant No. EP/ C546814/01. I acknowledge helpful correspondence with M. Wouters.
${ }^{1}$ J. Kasprzak et al., Nature (London) 443, 409 (2006).

${ }^{2}$ H. Deng, D. Press, S. Gotzinger, G. S. Solomon, R. Hey, K. H. Ploog, and Y. Yamamoto, Phys. Rev. Lett. 97, 146402 (2006).

${ }^{3}$ R. Balili, V. Hartwell, D. Snoke, L. Pfeiffer, and K. West, Science 316, 1007 (2007).

${ }^{4}$ M. S. Skolnick, T. A. Fisher, and D. M. Whittaker, Semicond. Sci. Technol. 13, 645 (1998).

${ }^{5}$ M. H. Szymanska, J. Keeling, and P. B. Littlewood, Phys. Rev. Lett. 96, 230602 (2006).

${ }^{6}$ M. Wouters and I. Carusotto, Phys. Rev. Lett. 99, 140402 (2007).

${ }^{7}$ J. Keeling and N. G. Berloff, Phys. Rev. Lett. (to be published).

${ }^{8}$ K. G. Lagoudakis, M. Wouters, M. Richard, A. Baas, I. Carusotto, R. André, L. S. Dang, and B. Deveaud-Plédran, arXiv:0801.1916 (unpublished).
${ }^{9}$ H. Vinck-Posada, B. A. Rodriguez, P. S. S. Guimaraes, A. Cabo, and A. Gonzalez, Phys. Rev. Lett. 98, 167405 (2007).

${ }^{10}$ P. R. Eastham, J. Phys.: Condens. Matter 19, 295210 (2007).

${ }^{11}$ D. Bajoni, P. Senellart, E. Wertz, I. Sagnes, A. Miard, A. Lemaître, and J. Bloch, Phys. Rev. Lett. 100, 047401 (2008).

${ }^{12}$ C. W. Lai et al., Nature (London) 450, 529 (2007).

${ }^{13}$ A. P. D. Love, D. N. Krizhanovskii, D. M. Whittaker, R. Bouchekioua, D. Sanvitto, S. Al Rizeiqi, M. S. Skolnick, P. R. Eastham, R. André, and L. S. Dang, Phys. Rev. Lett. (to be published).

${ }^{14}$ A. Baas, K. G. Lagoudakis, M. Richard, R. André, L. S. Dang, and B. Deveaud-Plédran, Phys. Rev. Lett. 100, 170401 (2008).

${ }^{15}$ I. A. Shelykh, Y. G. Rubo, G. Malpuech, D. D. Solnyshkov, and A. Kavokin, Phys. Rev. Lett. 97, 066402 (2006).

${ }^{16}$ G. Malpuech, D. D. Solnyshkov, H. Ouerdane, M. M. Glazov, 
and I. Shelykh, Phys. Rev. Lett. 98, 206402 (2007).

${ }^{17}$ A. Pikovsky, M. Rosenblum, and J. Kurths, Synchronization (Cambridge University Press, Cambridge, 2001).

${ }^{18}$ M. Wouters, Phys. Rev. B 77, 121302(R) (2008).

${ }^{19}$ D. Porras, C. Ciuti, J. J. Baumberg, and C. Tejedor, Phys. Rev. B 66, 085304 (2002).

${ }^{20}$ F. Tassone and Y. Yamamoto, Phys. Rev. B 59, 10830 (1999).

${ }^{21}$ G. Malpuech, A. Kavokin, A. Di Carlo, and J. J. Baumberg, Phys. Rev. B 65, 153310 (2002).
${ }^{22}$ D. Sarchi and V. Savona, Phys. Rev. B 75, 115326 (2007).

${ }^{23}$ P. R. Eastham and P. B. Littlewood, Phys. Rev. B 73, 085306 (2006).

${ }^{24}$ J. Keeling, F. M. Marchetti, M. H. Szymanska, and P. B. Littlewood, Semicond. Sci. Technol. 22, R1 (2007).

${ }^{25}$ M. O. Scully, Phys. Rev. Lett. 82, 3927 (1999).

${ }^{26}$ H. T. Cao, T. D. Doan, D. B. Tran Thoai, and H. Haug, Phys. Rev. B 69, 245325 (2004). 